\title{
Selection of Probiotics in the Prevention of Respiratory Tract Infections and Their Impact on Occupational Health: Scoping Review
}

\author{
José Antonio Picó-Monllor 1,*(D, Beatriz Ruzafa-Costas ${ }^{2}$, Eva Núñez-Delegido ${ }^{2}$, Pedro Sánchez-Pellicer ${ }^{2}$, \\ Javier Peris-Berraco ${ }^{3}$ and Vicente Navarro-Lopez ${ }^{2,4}$ (D) \\ 1 Department of Pharmacology, Pediatrics and Organic Chemistry, Faculty of Pharmacy, Universidad Miguel \\ Hernández de Elche, 03202 Elche, Spain \\ 2 MiBioPath Research Group, Health and Science Faculty, Catholic University of Murcia (UCAM), Campus de \\ los Jerónimos n 135, 30107 Murcia, Spain; beatriz.ruzafa@bioithas.com (B.R.-C.); \\ eva.nunez@bioithas.com (E.N.-D.); pedro.sanchez@bioithas.com (P.S.-P.); vnavarro@ucam.edu (V.N.-L.) \\ 3 Department R\&D, Korott, s.l., 03801 Alcoy, Spain; jperis@korott.com \\ 4 Clinical Microbiology and Infectious Disease Unit, Hospital Universitario Vinalopó, 03293 Elche, Spain \\ * Correspondence: japico@umh.es
}

Citation: Picó-Monllor, J.A.; Ruzafa-Costas, B.; Núñez-Delegido, E.; Sánchez-Pellicer, P.; Peris-Berraco,

J.; Navarro-Lopez, V. Selection of Probiotics in the Prevention of Respiratory Tract Infections and Their Impact on Occupational Health: Scoping Review. Nutrients 2021, 13, 4419. https://doi.org/10.3390/ nu13124419

Academic Editors: Javier Sanz-Valero and Elena Ronda

Received: 14 October 2021

Accepted: 8 December 2021

Published: 10 December 2021

Publisher's Note: MDPI stays neutral with regard to jurisdictional claims in published maps and institutional affiliations.

Copyright: (C) 2021 by the authors Licensee MDPI, Basel, Switzerland. This article is an open access article distributed under the terms and conditions of the Creative Commons Attribution (CC BY) license (https:/ / creativecommons.org/licenses/by/ $4.0 /)$.
Abstract: The occupational health impact of respiratory infectious diseases is costly to the economy and the health care system. Probiotics are non-pathogenic live microorganisms that, when ingested in adequate amounts, can colonize the intestinal tract, and enhance the immune system. In recent years, numerous studies have described the possible usefulness of certain probiotic strains in the treatment and prevention of respiratory tract infections, with disparate results. In order to assess the possible efficacy and safety of these microorganisms to prevent or ameliorate respiratory tract infections, we systematically searched the bibliographic databases MEDLINE (via Pubmed), EMBASE, The Cochrane library, Scopus, and Web of science, using the descriptors "Respiratory Tract Infections", "Probiotics", “Occupational Health", "Humans", and "Clinical Trials". After applying our inclusion and exclusion criteria, 18 studies were accepted for review and critical analysis. Our analysis suggests that a combination of different probiotics, most of them in the genus Bifidobacterium $\mathrm{sp}$. and Lactobacillus sp., could be a good mix to strengthen the immune system and reduce the symptoms of URTIs in the healthy working population.

Keywords: occupational health; probiotics; respiratory tract infections

\section{Introduction}

According to the $\mathrm{WHO}$, occupational health is an area of public health work whose mission is to promote and maintain the highest degree of physical, mental, and social wellbeing of workers in all occupations. Work-related health problems represent an economic loss of $4-6 \%$ of gross domestic product (GDP) in most countries [1].

Occupational health and safety contribute to reducing the costs of medical care, sick leave, and compensation for disability as they help avert the interruption of production processes and prevent occupational accidents and occupational diseases, as well as reducing labor passivity and absenteeism [2,3].

Chronic respiratory diseases, musculoskeletal disorders, noise-induced hearing loss, and skin problems are the most common occupational diseases. However, only a third of countries have programs to address these problems [1,4].

In 2006 in Beijing (Republic of China), the Global Alliance Against Chronic Respiratory Diseases (GARD) was created, led by the WHO. In its report on respiratory diseases and their impact worldwide, GARD describes the five respiratory diseases (chronic obstructive pulmonary disease, asthma, tuberculosis, lung cancer, and acute lower respiratory infections) that are among the most common causes of death all over the world. These 
are an immense health burden worldwide, and it is estimated that lower respiratory tract infections (LRTIs) cause almost 4 million deaths per year and are the leading cause of death among children under 5 years of age. Additionally, acute LRTIs in children set the stage for chronic respiratory diseases later in life. Moreover, those caused by the flu (influenza), kill 250,000 to 500,000 people and cost between USD 71 to 167 billion annually [5].

With these data, in 2017 the International Forum of Respiratory Societies (IFRS) wrote a report that exposes the tremendous impact that respiratory diseases have on world health and recommends, among other aspects, increasing research on the development of programs, tools, and strategies to prevent and better treat respiratory diseases by advocating for governmental (WHO) and non-governmental research organizations. Additionally, the IFRS promotes universal access to quality health care, including the availability of affordable essential medicines of guaranteed quality [6].

\subsection{Respiratory Tract Infections}

Respiratory tract infections (RTIs) owe their ethology to different viruses, such as rhinovirus, respiratory syncytial virus, influenza virus, human parainfluenza virus, human metapneumovirus, paramyxovirus, mycovirus, adenovirus and coronavirus, or bacteria such as Streptococcus pneumoniae, Mycoplasma pneumoniae, Haemophiles influenzae, Chlamydia pneumoniae, Coxiella burnetii, and Legionella pneumophila.

Infections of the respiratory system can affect the upper respiratory tract, which includes the nose, the paranasal sinuses, the pharynx, and larynx, as well as the lower respiratory tract, formed by the trachea, the bronchi, the bronchioles, and lungs. Upper respiratory tract infections (URTIs) cause tonsillitis, pharyngitis, sinus laryngitis, otitis media, certain types of flu, and the common cold, whereas LRTIs lead to more serious diseases such as pneumonia [7,8].

\subsection{Probiotics}

Probiotics are live microorganisms that, when administered in adequate amounts, confer a health benefit on the host [9]. Recently, the International Scientific Association for Probiotics \& Prebiotics [10] established a consensus document with a series of criteria for microorganisms that are components of products aimed at the consumer to be considered probiotics:

- The microorganism in question must have been scientifically proven to be a safe species that is supported by sufficient evidence of general beneficial effect in humans.

- Evidence of its viability as a microorganism should be available in human studies conducted.

Recent meta-analyses and systematic reviews support the health benefits of probiotics in relation to the modulatory effect on the immune system, as reported in the study by Miller et al. [11] showing positive results in cellular immune function responsible for firstline defense against pathogenic microorganisms, or the work by Hajavi et al. [12], in which probiotics could increase systemic interferon (IFN), interleukin 10 (IL-10), and interleukin 12 (IL-12) production, elevate pro-Th1 cytokine immune responses, and decrease the profile of elevated Th2 cytokines in allergic diseases.

Darbandi et al. [8] and Hao et al. [13] in their studies suggest that probiotics are a complementary treatment in diseases of the respiratory system and a viable option to promote a faster recovery from such diseases. On the other hand, the study by Mahooti et al. [14], suggests that, due to the antiviral properties of probiotics against other viruses, they could be a complementary treatment alternative against SARS CoV-2.

In summary, supplementation with probiotics, prebiotics, and synbiotics has shown promising results against several enteric pathogens due to their unique ability to compete with the pathogenic microbiota, to kill it or to stimulate, modulate, and regulate the immune response of the host by initiating the activation of specific genes within and outside the intestinal tract of the host $[15,16]$.

By virtue of their benefits, probiotic products can contribute significantly to the health of the population and, therefore, generate less expenditure for health systems. The works 
of Lenoir-Wijnkoop et al. $[17,18]$ conducted in France and the USA, respectively, evaluated the impact on public health and the cost of the use of probiotics in RTIs. In the French study, it was found that the widespread use of probiotics could eliminate the equivalent of 2.4 million days of RTIs, 581,000 days off work, and 291,000 antibiotic treatments, which would generate health savings of between EUR 14.6 and 37.7 million per year. The study in the USA presented similar conclusions.

There are other systematic reviews and meta-analyses in which the results show a positive association between synbiotic [7] or probiotic $[8,13,14]$ consumption and the prevention of RTIs in different populations. However, we have not found reviews in which this association is made with specific probiotic strains in a healthy working population.

Considering the benefits of certain probiotics in diseases of the respiratory system and their ability to stimulate the immune response of the host, we proposed this scoping review to identify and select potential probiotic strains to prevent URTIs, decrease the severity in those cases that end up developing the disease, and diminish their impact on occupational health.

\section{Materials and Methods}

\subsection{Design}

A descriptive study and critical analysis of works retrieved by scoping review according to Preferred Reporting Items for Systematic reviews and Meta-Analyses (PRIS-MA.

\subsection{Data Collection Source}

This review aimed to carry out a critical and systematic study of the works published in different databases through direct consultation and access via the Internet to works collected in the following databases: MEDLINE (via PubMed), EMBASE, SCOPUS, Cochrane Library Plus, and Institute for Scientific Information (ISI)-Web of Science.

\subsection{Information Processing}

To define the documentary search, the Thesaurus developed by the US National Library of Medicine (Medical Subjects Headings-Mesh) was used. Entry terms were also used. The terms "Occupational health", "Probiotics", and "Respiratory tract Infections" were used as descriptors and free text in titles and abstracts. The final search equation was developed for use in the MEDLINE database, via PubMed, by using the Boolean connectors and the "Humans" and "Clinical Trial" filters, with the following results:

("Respiratory Tract Infections" [Title/Abstract] OR “Respiratory Infections" [Title/ Abstract] OR "Upper Respiratory Infections" [Title/Abstract] OR "Respiratory Tract Infections" [MeSH Terms]) AND ("Microbiota" [MeSH Terms] OR "microbiota*” [Title/ Abstract] OR "Microbial Community" [Title/Abstract] OR "Microbial Communities" [Title/Abstract] OR "microbiome*" [Title/Abstract] OR "Microbial Flora" [Title/Abstract] OR "Microflora" [Title/Abstract] OR "Dysbiosis" [MeSH Terms] OR "Dysbiosis" [Title/ Abstract] OR "Disbiosis" [Title/Abstract] OR "Dysbacteriosis" [Title/Abstract] OR "Probiotics" [MeSH Terms] OR "Probiotics" [Title/Abstract] OR "Dietary Supplements" [Title/Abstract]) OR "Occupational Health" [Mesh] OR "Occupational Health" [Title/Abstract] OR "Industrial Hygiene" [Title/Abstract] OR "Industrial Health" [Title/Abstract] OR "Occupational Safety" [Title/Abstract] OR "Employee Health" [Title/Abstract] OR "Occupational Risks" [Title/Abstract] OR “Insecure Labor Conditions" [Title/Abstract] OR "Occupational Risk" [Title/Abstract] OR “Work Risk" [Title/Abstract] OR “Occupational Hazard" [Title/Abstract] OR "Risk at Work" [Title/Abstract] OR "Professional Health" [Title/Abstract] OR "Working Conditions" [Title/Abstract] OR “Occupational Stress" [Mesh] OR "Job Stress" [Title/Abstract] OR "Professional Stress" [Title/Abstract] OR "Work Place Stress" [Title/Abstract] OR "Workplace Stress" [Title/Abstract] OR "Work Medicine" [Title/Abstract].

According to the different characteristics of the rest of the databases mentioned, the same strategy was adopted. The search was carried out from the first available date, until 
August 2021 (time of the last update). Additionally, as a secondary search and to reduce the number of papers not retrieved, the bibliographic list of articles that were selected in the main search was examined, in order to identify studies not detected in the review.

\subsection{Final Selection of Articles}

The final selection of the articles was made based on the following inclusion criteria: The papers had to be original clinical studies published in peer-reviewed journals, show a causal relationship between the intake of "probiotics" in "healthy subjects of a working age" and "respiratory tract infections", selecting those pertinent whose full text could be retrieved, and be written in English, Portuguese, or Spanish. Those not carried out in humans or that did not focus the intervention on probiotics, on healthy people over 19 years old, and/or on RTIs were excluded.

The selection of the relevant articles was carried out independently by the authors of the present review (J.A.P.-M., B.R.-C., P.S.-P., E.N.-D., J.P.-B. and V.N.-L). To validate the choice of articles for the review, it was established that the assessment of concordance between two of these authors (Kappa index) should be greater than 0.80 (a measure of the strength of a very good agreement). Provided that this condition was met, possible disagreements would be resolved by consulting an expert in the field and subsequent consensus among the authors [19].

\subsection{Assessment of Methodological Quality}

The quality of the selected articles was assessed jointly, taking as support the guidelines for the communication of clinical studies, Consolidated Standards of Reporting Trials (CONSORT) [20], which contains a list of 25 essential points that should be described in the publication of these studies. For each selected article, a point was assigned for each item depending on whether the information was (" 1 ") or was not (" 0 ") collected in the article. In case the evaluation of any item was not necessary, that point was not counted in the total (not applicable $=\mathrm{NA}$ ). When an item was composed of several points, these were evaluated independently, giving the same value to each of them, and later an average was calculated (this being the result for that item), in such a way that in no case could the score per item exceed 1 point.

\subsection{Data Extraction}

The control of the information extracted from the reviewed studies was carried out by means of double-entry tables that allowed the detection of errors and correction by re-consulting the originals.

To determine the validity of the articles, the Burton-Kebler half-period (the median age) and the Price index (percentage of articles aged less than 5 years) were calculated. The articles were grouped according to the variables under study, to systematize and facilitate the understanding of the results, coding the following data: first author of the bibliographic reference and year of publication, study design, country where the study was carried out, number of participants, study population, period in which the work was carried out, what type of intervention took place, and the results obtained.

\section{Results}

Using the search criteria described, 2338 references were retrieved: $1285(54.96 \%)$ in MEDLINE, 6 in EMBASE (0.26\%), 5 in Scopus (0.21\%), 314 in the Cochrane Library (13.4\%), and 728 in Web of Science (31.1\%). After cleaning the duplicates, applying the inclusion and exclusion criteria, and consulting the bibliographic lists of the selected articles (Figure 1), it was possible to select 18 documents for review and critical analysis (Table 1). 

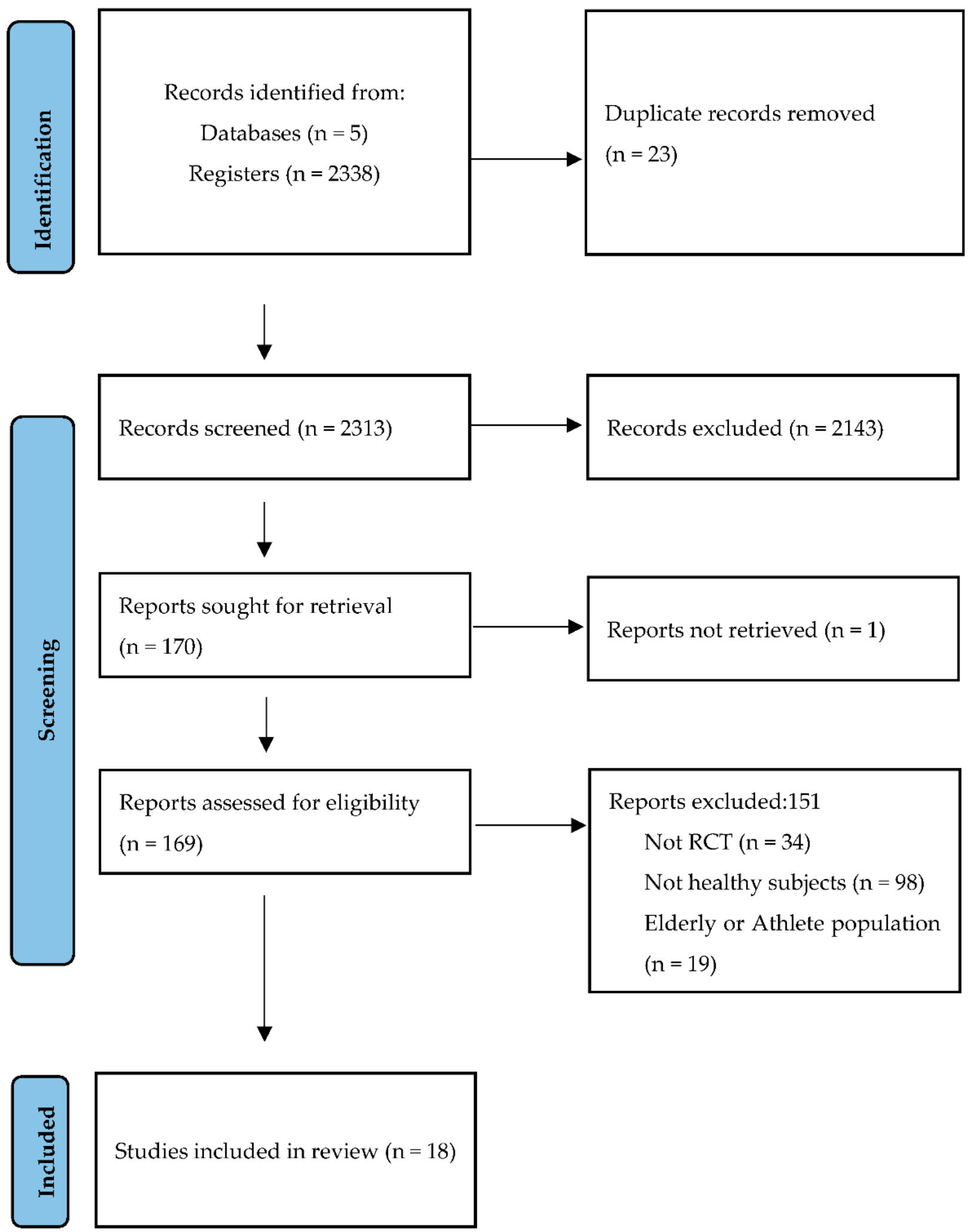

Figure 1. Identification and selection of studies according to Preferred Reporting Items for Systematic Reviews and Meta-Analyses (PRISMA statement) [21]. 
Table 1. Summary of reviewed studies on the relationship between probiotics and respiratory tract infections in healthy subjects.

\begin{tabular}{|c|c|c|c|c|c|c|c|}
\hline Author (Year) & Design & Country & Participants & Population & Monitoring & Intervention Performed & Results \\
\hline Ahrén (2021) [22] & $\begin{array}{l}\text { Randomized, } \\
\text { double-blind, } \\
\text { placebo-controlled } \\
\text { trial multicentric }\end{array}$ & Germany & $\begin{array}{c}898 \\
\text { M/F: } 322 / 576 \\
\text { Median age: } 40.6 \\
\text { years }\end{array}$ & $\begin{array}{l}\text { Healthy men and } \\
\text { women }\end{array}$ & 3 years & $\begin{array}{l}\text { Lactiplantibacillus plantarum } \\
\text { HEAL9 and } \\
\text { Lacticaseibacillus paracasei } \\
8700: 2\left(1 \times 10^{9} \mathrm{cfu}\right)\end{array}$ & $\begin{array}{l}\text { Protects against multiple } \\
\text { colds in adults prone to } \\
\text { getting colds. }\end{array}$ \\
\hline Zhang (2021) [23] & $\begin{array}{l}\text { Randomized, } \\
\text { double-blinded } \\
\text { placebo-controlled } \\
\text { clinical trial }\end{array}$ & China & $\begin{array}{c}123 \\
\text { M/F: } 69 / 54 \\
\text { Median age } 37.2 \\
\text { years }\end{array}$ & $\begin{array}{l}\text { Healthy } \\
\text { white-collar } \\
\text { workers }\end{array}$ & 12 weeks & $\begin{array}{l}\text { Qingrun yogurt with } \\
\text { Bifidobacterium animalis } \\
\text { subsp. lactis Bl-04 }\left(2 \times 10^{9}\right. \\
\text { cfu), Lactobacillus casei, } \\
\text { Lactobacillus bulgaricus and } \\
\text { Streptococcus thermophilus }\end{array}$ & $\begin{array}{l}\text { Qingrun yogurt was able } \\
\text { to reduce the incidence, } \\
\text { duration, and severity of } \\
\text { URTIs and improve } \\
\text { immune biomarkers. }\end{array}$ \\
\hline Wang (2021) [24] & $\begin{array}{l}\text { Randomized } \\
\text { controlled clinical } \\
\text { trial: Study pilot. } \\
\text { Multicentric }\end{array}$ & China & $\begin{array}{c}193 \\
\text { M/F: } 56 / 137 \\
\text { Median age: } 36.1 \\
\text { years }\end{array}$ & Healthcare workers & 1 month & $\begin{array}{l}\text { Streptococcus thermophilus } \\
\text { ENT-K12 }\end{array}$ & $\begin{array}{l}\text { ENT-K12 creates a stable } \\
\text { URT microbiota for at least } \\
20 \text { days and protects } \\
\text { medical staff from URTIs, } \\
\text { can reduce the length of } \\
\text { sick days, days off work, } \\
\text { and days on antibiotics } \\
\text { and antivirals. }\end{array}$ \\
\hline $\begin{array}{c}\text { Kinoshita (2019) } \\
\text { [26] }\end{array}$ & $\begin{array}{l}\text { Randomized } \\
\text { controlled } \\
\text { open-label study }\end{array}$ & Japan & $\begin{array}{c}\text { F: } 961 \\
\text { Median age: } 39.3 \\
\text { years }\end{array}$ & $\begin{array}{l}\text { Women health } \\
\text { workers }\end{array}$ & 16 weeks & $\begin{array}{l}\text { Lactobacillus delbrueckii } \\
\text { subsp. bulgaricus } \\
\text { (OLL1073R-1) and } \\
\text { Streptococcus thermophilus } \\
\left(1 \times 10^{9} \mathrm{cfu}\right)\end{array}$ & $\begin{array}{l}\text { The probiotic mixture did } \\
\text { not show any prevention } \\
\text { of influenza and NK cell } \\
\text { activity enhancement. } \\
\text { However, a significant } \\
\text { increase in IFN- } \gamma \\
\text { production was found. }\end{array}$ \\
\hline
\end{tabular}


Table 1. Cont.

\begin{tabular}{|c|c|c|c|c|c|c|c|}
\hline Author (Year) & Design & Country & Participants & Population & Monitoring & Intervention Performed & Results \\
\hline Zhang (2018) [27] & $\begin{array}{l}\text { Randomized } \\
\text { double-blind } \\
\text { placebo-controlled } \\
\text { prospective trial. } \\
\text { Single center }\end{array}$ & China & $\begin{array}{c}134 \\
\text { M/F: } 66 / 68 \\
\text { Median age: } 34.3 \\
\text { years }\end{array}$ & Healthy subjects & 12 weeks & $\begin{array}{l}\text { Lactobacillus paracasei } \geq 3 \\
\times 10^{7} \mathrm{cfu}, \text { L. casei } 431^{\circledR} \geq 3 \\
\times 10^{7} \mathrm{cfu} \text { and Lactobacillus } \\
\text { fermentum } \mathrm{PCC}^{\circledR} \geq 3 \times 10^{6} \\
\mathrm{cfu}\end{array}$ & $\begin{array}{l}\text { Mix probiotics were safe } \\
\text { and effective for fighting } \\
\text { the common cold and } \\
\text { influenza-like respiratory } \\
\text { infections by boosting the } \\
\text { immune system. } \\
\text { LCZ alleviated URTI }\end{array}$ \\
\hline Hor (2018) [28] & $\begin{array}{l}\text { Randomized, } \\
\text { double-blind and } \\
\text { placebo-controlled } \\
\text { study }\end{array}$ & Malaysia & $\begin{array}{c}137 \\
\text { M/F: } 62 / 75 \\
\text { Median age: } 44.2 \\
\text { years }\end{array}$ & $\begin{array}{l}\text { Healthy adults and } \\
\text { elderly }\end{array}$ & 12 months & $\begin{array}{l}\text { Lactobacillus casei Zhang } \\
\left(10^{9} \mathrm{cfu}\right)\end{array}$ & $\begin{array}{l}\text { symptoms in adults, with } \\
\text { peduced duration for nasal, } \\
\text { pharyngeal, general flu, } \\
\text { and total respiratory } \\
\text { illness symptoms } \\
\text { compared to the placebo. } \\
\text { The timing of BB-12 }\end{array}$ \\
\hline Meng (2016) [37] & $\begin{array}{l}\text { Randomized, } \\
\text { partially blinded, } \\
\text { four-period } \\
\text { crossover study }\end{array}$ & USA & $\begin{array}{c}30 \\
\text { M/F: } 11 / 29 \\
\text { Median age: } 28 \\
\text { years }\end{array}$ & $\begin{array}{l}\text { Healthy } \\
\text { subjects }\end{array}$ & 4 weeks & $\begin{array}{l}\text { (i) YS } \\
\text { (ii) YS with BB-12 }{ }^{\circledR} \text { added } \\
\text { prefermentation }(\mathrm{PRE}) \text {; } \\
\text { (iii) YS with BB-12 }{ }^{\circledR} \text { added } \\
\text { postfermentation }(\mathrm{POST}) \\
\text { (iv) one capsule BB-12 }{ }^{\circledR}\end{array}$ & $\begin{array}{l}\text { addition to yogurt } \\
\text { smoothies in relation to the } \\
\text { fermentation process } \\
\text { influenced the impact of } \\
\text { BB-12 on immune function } \\
\text { and cold/flu severity in } \\
\text { young healthy adults. }\end{array}$ \\
\hline $\begin{array}{c}\text { Jespersen (2015) } \\
\text { [29] }\end{array}$ & $\begin{array}{l}\text { Randomized } \\
\text { double-blind, } \\
\text { placebo-controlled } \\
\text { trial multicentric }\end{array}$ & Denmark/Germany & $\begin{array}{c}1104 \\
\text { M/F: } 453 / 651 \\
\text { Median age: } 31.6 \\
\text { years }\end{array}$ & $\begin{array}{l}\text { Healthy men and } \\
\text { women with } \\
\text { influenza } \\
\text { vaccination }\end{array}$ & 42 days & L. casei $431^{\circledR}\left(\geq 10^{9} \mathrm{cfu}\right)$ & $\begin{array}{l}\text { L. casei } 431 \text { did not show } \\
\text { an effect on antibody titers } \\
\text { and influenza A-specific } \\
\text { antibodies } 3 \text { weeks after } \\
\text { influenza vaccination but } \\
\text { may reduce the duration of } \\
\text { common cold and ILI } \\
\text { episodes in healthy adults. }\end{array}$ \\
\hline Shida (2015) [30] & $\begin{array}{l}\text { Randomized } \\
\text { controlled trial }\end{array}$ & Japan & $\begin{array}{c}\text { M: } 100 \\
\text { Median age: } 40.6 \\
\text { years }\end{array}$ & $\begin{array}{l}\text { Healthy male } \\
\text { workers }\end{array}$ & 4 months & $\begin{array}{l}\text { Lactobacillus casei Shirota }{ }^{\circledR} \\
\left(1.0 \times 10^{11} \mathrm{cfu}\right) \text { fermented } \\
\text { milk }\end{array}$ & $\begin{array}{l}\text { Fermented milk with LcS } \\
\text { may reduce the risk of } \\
\text { URTIs in healthy } \\
\text { middle-aged office } \\
\text { workers. }\end{array}$ \\
\hline
\end{tabular}


Table 1. Cont.

\begin{tabular}{|c|c|c|c|c|c|c|c|}
\hline Author (Year) & Design & Country & Participants & Population & Monitoring & Intervention Performed & Results \\
\hline Smith (2012) [38] & $\begin{array}{l}\text { Randomized } \\
\text { double blind } \\
\text { placebo-controlled } \\
\text { trial }\end{array}$ & USA & $\begin{array}{c}198 \\
\text { M/F: } 47 / 151 \text { Age: } \\
\text { 19 to } 25 \text { years }\end{array}$ & $\begin{array}{l}\text { Healthy } \\
\text { subjects }\end{array}$ & 12 weeks & $\begin{array}{l}\text { Lactobacillus rhamnosus } \\
\text { LGG }^{\circledR} \text { and Bifidobacterium } \\
\text { animalis ssp. lactis BB- } 12^{\circledR} \\
\left(1 \times 10^{9} \mathrm{cfu}\right)\end{array}$ & $\begin{array}{l}\text { Mix probiotics LGG }{ }^{\circledR} \text { and } \\
\text { BB- } 12^{\circledR} \text { may be beneficial } \\
\text { for mitigating decrements } \\
\text { in HRQL during URTI in } \\
\text { college students living on } \\
\text { campus in residence halls. }\end{array}$ \\
\hline West (2013) [39] & $\begin{array}{l}\text { Randomized } \\
\text { double-blind } \\
\text { placebo-controlled } \\
\text { parallel trial }\end{array}$ & Australia & $\begin{array}{c}465 \\
\text { M/F: } 241 / 224 \\
\text { Median age: } 36 \\
\text { years }\end{array}$ & $\begin{array}{l}\text { Healthy } \\
\text { subjects }\end{array}$ & 160 days & $\begin{array}{l}\text { Bifidobacterium animalis } \\
\text { subsp. lactis Bl-04 }\left(2 \times 10^{9}\right. \\
\text { cfu), Lactobacillus } \\
\text { acidophilus NCFM and } \\
\text { Bifidobacterium animalis } \\
\text { subsp. lactis Bi-07 (NCFM } \\
\mathcal{E} \text { Bi-07) }\left(5 \times 10^{9} \mathrm{cfu}\right)\end{array}$ & $\begin{array}{l}\text { Bl-04 appears to be a } \\
\text { useful nutritional } \\
\text { supplement in reducing } \\
\text { the risk of URTI in healthy, } \\
\text { physically active adults. }\end{array}$ \\
\hline $\begin{array}{c}\text { Rizzardini (2011) } \\
\text { [31] }\end{array}$ & $\begin{array}{l}\text { Randomized, } \\
\text { double-blind, } \\
\text { placebo controlled, } \\
\text { four-arm, } \\
\text { parallel-group } \\
\text { study }\end{array}$ & Italy & $\begin{array}{c}211 \\
\text { M/F: } 93 / 118 \\
\text { Median age: } 29 \text { and } \\
\text { 37.3 years }\end{array}$ & $\begin{array}{l}\text { Healthy adults } \\
\text { with influenza } \\
\text { vaccination }\end{array}$ & 6 weeks & $\begin{array}{l}\text { BB- } 12^{\circledR} \text { capsule or } L \text {. casei } \\
431^{\circledR} \text { drink }\left(1 \times 10^{9} \text { cfu }\right) \\
\text { and Fluad }^{\circledR}\end{array}$ & $\begin{array}{l}\text { BB-12 or L. casei } 431^{\circledR} \\
\text { significantly increases } \\
\text { antigen-specific immune } \\
\text { responses in healthy } \\
\text { individuals receiving an } \\
\text { influenza vaccination. }\end{array}$ \\
\hline $\begin{array}{c}\text { Davidson (2011) } \\
\text { [32] }\end{array}$ & $\begin{array}{l}\text { Randomized } \\
\text { double-blind } \\
\text { placebo-controlled } \\
\text { pilot study }\end{array}$ & USA & $\begin{array}{c}42 \\
\text { M/F: } 16 / 26 \\
\text { Median age: } 33.5 \\
\text { years }\end{array}$ & $\begin{array}{l}\text { Healthy adults } \\
\text { with LAIV }\end{array}$ & 28 days & $\begin{array}{l}\text { Lactobacillus GG }\left(1 \times 10^{10}\right. \\
\mathrm{cfu}) \text { and LAIV }\end{array}$ & $\begin{array}{l}\text { Lactobacillus } \mathrm{GG}^{\circledR} \text { : is } \\
\text { potentially an important } \\
\text { adjuvant to improve } \\
\text { influenza vaccine } \\
\text { immunogenicity. }\end{array}$ \\
\hline $\begin{array}{c}\text { Guillemard (2010) } \\
\text { [33] }\end{array}$ & $\begin{array}{l}\text { Randomized, } \\
\text { double-blind, } \\
\text { controlled study } \\
\text { Single-center }\end{array}$ & Germany & $\begin{array}{c}1000 \\
\text { M/F: } 220 / 780 \\
\text { Median age: } 31.8 \\
\text { years }\end{array}$ & $\begin{array}{l}\text { Healthy men and } \\
\text { women }\end{array}$ & $\begin{array}{l}4 \text { months and } 2 \\
\text { weeks }\end{array}$ & $\begin{array}{l}\text { Verum: Lactobacillus casei } \\
\text { DN-114 } 001\left(1 \times 10^{10} \mathrm{cfu}\right) \text {, } \\
\text { Streptococcus thermophilus, } \\
\text { Lactobacillus delbrueckii } \\
\text { subsp. bulgaricus }\end{array}$ & $\begin{array}{l}\text { Verum consumption was } \\
\text { associated with significant } \\
\text { improvement in incidence } \\
\text { of CIDs in shift workers. }\end{array}$ \\
\hline $\begin{array}{c}\text { Berggren (2010) } \\
{[34]}\end{array}$ & $\begin{array}{l}\text { Randomized, } \\
\text { double-blind, } \\
\text { placebo-controlled } \\
\text { trial multicentric }\end{array}$ & Sweden & $\begin{array}{c}272 \\
\text { M/F: } 92 / 180 \\
\text { Median age: } 46.5 \\
\text { years }\end{array}$ & $\begin{array}{l}\text { Healthy men and } \\
\text { women }\end{array}$ & 12 weeks & $\begin{array}{l}\text { Lactiplantibacillus plantarum } \\
\text { HEAL9 and } \\
\text { Lacticaseibacillus paracasei } \\
8700: 2\left(1 \times 10^{9}\right) \mathrm{cfu}\end{array}$ & $\begin{array}{l}\text { Contributes to the body's } \\
\text { defense against common } \\
\text { cold infections } p<0.05 \text {. }\end{array}$ \\
\hline
\end{tabular}


Table 1. Cont.

\begin{tabular}{|c|c|c|c|c|c|c|c|}
\hline Author (Year) & Design & Country & Participants & Population & Monitoring & Intervention Performed & Results \\
\hline De Vrese (2005) [36] & $\begin{array}{l}\text { Randomized, } \\
\text { double-blind, } \\
\text { placebo-controlled } \\
\text { intervention study }\end{array}$ & Germany & $\begin{array}{c}479 \\
\text { M/F: } 185 / 294 \\
\text { Median age: } 37 \\
\text { years }\end{array}$ & $\begin{array}{l}\text { Healthy men and } \\
\text { women }\end{array}$ & 8.5 months & $\begin{array}{l}\text { Lactobacillus gasseri PA } \\
16 / 8, \text { Bifidobacterium } \\
\text { longum SP } 07 / 3 \text {, } \\
\text { Bifidobacterium bifidum } \mathrm{MF} \\
20 / 5\left(5 \times 10^{7} \mathrm{cfu}\right) \text { (Tribion } \\
\left.\text { harmonis }^{\mathrm{TM}}\right)\end{array}$ & $\begin{array}{l}\text { The intake of mix probiotic } \\
\text { for at least } 3 \text { months } \\
\text { significantly shortened } \\
\text { common cold episodes by } \\
\text { almost } 2 \text { days and reduced } \\
\text { the severity of symptoms. }\end{array}$ \\
\hline Tubelius (2005) [35] & $\begin{array}{l}\text { Randomized, } \\
\text { double-blind } \\
\text { placebo-controlled } \\
\text { study }\end{array}$ & Sweden & $\begin{array}{c}181 \\
\text { M/F: } 65 / 71 \\
\text { Median age: } 44 \\
\text { years }\end{array}$ & Healthy workers & 80 days & $\begin{array}{l}\text { Lactobacillus reuteri } \\
\text { protectis (ATCC55730) } 1 \times \\
10^{8} \mathrm{cfu}\end{array}$ & $\begin{array}{l}\text { Lactobacillus reuteri is } \\
\text { effective to promote } \\
\text { workplace healthiness. In } \\
\text { the studied population, } \\
\text { sick-days caused by } \\
\text { respiratory or } \\
\text { gastrointestinal diseases } \\
\text { could be reduced by } 55 \% \text {. }\end{array}$ \\
\hline
\end{tabular}

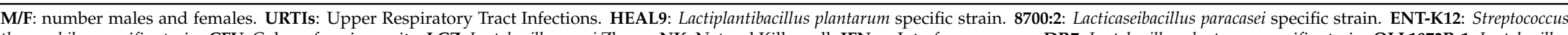

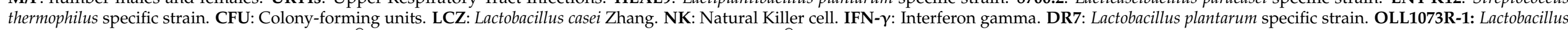

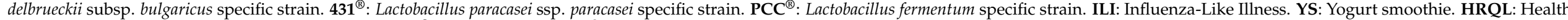

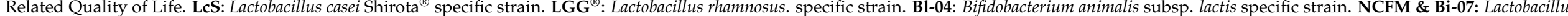

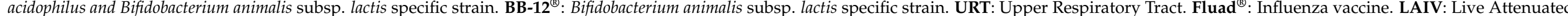

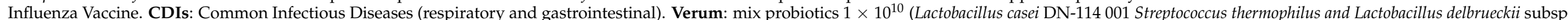
Bulgaricus). Tribion harmonis ${ }^{\mathrm{TM}}$ : mix probiotics Lactobacillus gasseri PA 16/8, Bifidobacterium longum SP 07/3, Bifidobacterium bifidum MF $20 / 5$ specific strains. 
The agreement on the relevance of the selected studies among all evaluators, calculated using the Kappa index, was $81 \%$.

The chosen articles presented an obsolescence, according to the Burton-Kebler Index (IBK), equal to 6 years, with a Price Index (IP) of 38.9\%. Pearson's coefficient was $\mathrm{r}^{2}=0.06$. The years with the highest number of published works were 2021 with three [22-24] followed by two studies each in 2019 [25,26], 2018 [27,28], 2015 [29,30], 2011 [31,32], 2010 [33,34], and 2005 [35,36]. The years with only one publication were 2016 [37], 2012 [38], and 2013 [39].

When performing the CONSORT questionnaire, the scores ranged from a minimum of 13 (out of 25 items) to a maximum of 21.5 (out of 21 items), with a median equal to 18.5 (Table 2).

Table 2. Assessment of the methodological quality of the studies analyzed by means of the 25 items of the CONSORT $2010[20]$.

\begin{tabular}{|c|c|c|c|c|c|c|c|c|c|c|c|c|c|c|c|c|c|c|c|c|c|c|c|c|c|c|c|}
\hline & 1 & 2 & 3 & 4 & 5 & 6 & 7 & 8 & 9 & 10 & 11 & 12 & 13 & 14 & 15 & 16 & 17 & 18 & 19 & 20 & 21 & 22 & 23 & 24 & 25 & $\mathbf{T}$ & $\%$ \\
\hline Ahrén [22] & 1 & 1 & 1 & 1 & 1 & 1 & 0.5 & 1 & 0 & 1 & 1 & 1 & 1 & 0.5 & 1 & 1 & 0.5 & NA & 1 & 1 & 1 & 1 & 1 & 1 & 1 & 21.5 & 90 \\
\hline Zhang [23] & 1 & 1 & 1 & 1 & 1 & 0.5 & 0 & 0 & 0 & 1 & 1 & 0.5 & 1 & 0.5 & 1 & 1 & 0.5 & NA & 0 & 1 & 1 & 1 & 1 & 1 & 1 & 18 & 75 \\
\hline Wang [24] & 0.5 & 1 & 1 & 1 & 1 & 0.5 & 0 & 0 & 0 & 1 & 1 & 0.5 & 1 & 0.5 & 1 & 1 & 0.5 & NA & 1 & 1 & 1 & 1 & 1 & 1 & 1 & 18.5 & 77 \\
\hline Chong [25] & 1 & 1 & 1 & 1 & 1 & 0.5 & 0.5 & 0.5 & 0 & 0 & 0 & 0.5 & 1 & 0.5 & 1 & 1 & 1 & NA & 1 & 0 & 1 & 1 & 0 & 0 & 1 & 15.5 & 65 \\
\hline $\begin{array}{c}\text { Kinoshita } \\
\text { [26] }\end{array}$ & 1 & 1 & 1 & 1 & 1 & 0.5 & 0.5 & 1 & 1 & 1 & 1 & 0.5 & 1 & 0.5 & 1 & 1 & 0.5 & NA & 1 & 1 & 1 & 1 & 1 & 1 & 1 & 21.5 & 90 \\
\hline Zhang [27] & 0.5 & 1 & 1 & 1 & 1 & 0.5 & 0.5 & 1 & 1 & 1 & 1 & 0.5 & 1 & 0.5 & 1 & 1 & 0.5 & NA & 1 & 1 & 1 & 1 & 1 & 1 & 1 & 21 & 87 \\
\hline Hor [28] & 0 & 1 & 1 & 0.5 & 1 & 0.5 & 0 & 1 & 1 & 1 & 0 & 0.5 & 0 & 0.5 & 1 & 0.5 & 1 & NA & 0 & 0 & 1 & 1 & 0 & 0 & 1 & 13 & 54 \\
\hline Meng [37] & 0.5 & 1 & 1 & 1 & 1 & 0.5 & 0.5 & 1 & 1 & 1 & 0.5 & 0.5 & 1 & 0.5 & 1 & 1 & 0.5 & NA & 1 & 1 & 1 & 1 & 1 & 1 & 1 & 20.5 & 85 \\
\hline $\begin{array}{c}\text { Jespersen } \\
\text { [29] }\end{array}$ & 1 & 1 & 1 & 1 & 1 & 0.5 & 0.5 & 1 & 1 & 1 & 0.5 & 1 & 1 & 0.5 & 1 & 1 & 0.5 & NA & 1 & 1 & 1 & 1 & 1 & 1 & 1 & 21.5 & 90 \\
\hline Shida [30] & 0.5 & 1 & 1 & 1 & 1 & 0.5 & 0.5 & 0.5 & 0 & 1 & 0 & 0.5 & 1 & 0.5 & 1 & 1 & 0.5 & NA & 1 & 0 & 1 & 1 & 1 & 1 & 1 & 17.5 & 73 \\
\hline Smith [38] & 0.5 & 1 & 1 & 1 & 1 & 0.5 & 0.5 & 1 & 1 & 1 & 0.5 & 0.5 & 1 & 0.5 & 1 & 1 & 0.5 & NA & 1 & 1 & 1 & 1 & 1 & 1 & 1 & 20.5 & 85 \\
\hline West [39] & 0.5 & 1 & 1 & 1 & 1 & 1 & 0.5 & 1 & 0 & 0 & 0 & 0.5 & 1 & 0.5 & 1 & 1 & 0.5 & NA & 1 & 0 & 1 & 1 & 1 & 1 & 1 & 17.5 & 73 \\
\hline $\begin{array}{c}\text { Rizzardini } \\
{[31]}\end{array}$ & 1 & 1 & 1 & 1 & 1 & 0.5 & 0.5 & 1 & 1 & 1 & 0.5 & 0.5 & 1 & 0.5 & 1 & 1 & 0.5 & NA & 1 & 1 & 1 & 1 & 1 & 1 & 1 & 21 & 87 \\
\hline $\begin{array}{c}\text { Davidson } \\
\text { [32] }\end{array}$ & 1 & 1 & 1 & 1 & 1 & 0.5 & 0.5 & 0.5 & 0 & 1 & 0.5 & 0.5 & 1 & 0.5 & 1 & 1 & 0.5 & NA & 1 & 1 & 1 & 1 & 1 & 1 & 1 & 19.5 & 81 \\
\hline $\begin{array}{l}\text { Guillemard } \\
\text { [33] }\end{array}$ & 1 & 1 & 1 & 1 & 1 & 0.5 & 0.5 & 1 & 1 & 1 & 0.5 & 0.5 & 1 & 0.5 & 1 & 1 & 0.5 & NA & 1 & 0 & 1 & 1 & 0 & 0 & 1 & 18 & 75 \\
\hline $\begin{array}{c}\text { Berggren } \\
{[34]}\end{array}$ & 1 & 1 & 0.5 & 1 & 1 & 0.5 & 0.5 & 0.5 & 0 & 0 & 0.5 & 0.5 & 1 & 0.5 & 1 & 1 & 0.5 & NA & 1 & 0 & 1 & 1 & 0 & 0 & 1 & 15 & 62 \\
\hline $\begin{array}{c}\text { De Vrese } \\
{[36]}\end{array}$ & 1 & 1 & 1 & 1 & 0 & 0.5 & 0.5 & 0.5 & 1 & 1 & 0.5 & 0.5 & 1 & 0.5 & 1 & 1 & 0.5 & NA & 1 & 0 & 1 & 1 & 0 & 0 & 1 & 16.5 & 69 \\
\hline $\begin{array}{c}\text { Tubelius } \\
\text { [35] }\end{array}$ & 1 & 1 & 1 & 1 & 1 & 0.5 & 0 & 0 & 0 & 1 & 0.5 & 0.5 & 0.5 & 0 & 1 & 0 & 0 & NA & 1 & 1 & 1 & 1 & 1 & 1 & 1 & 16 & 67 \\
\hline
\end{tabular}

Most of the studies (88\%) included in the review were double-blind clinical trials with placebo and control group [22-29,31-39], except for Shida et al. [30] without masking patients and researchers. Of these works, four were developed in Germany $[22,29,33,36]$, three each in China [23,24,27] and the United States [32,37,38], two each in Malaysia [25,28], Japan [26,30], and Sweden [34,35], and a single study each in the countries of Denmark [29], Australia [39], and Italy [31]. All of them written in English.

According to the number of participants, the studies that presented the smallest sample size were Meng et al. [37] and Davidson et al. [32] with 30 and 40 people, respectively, while the works that presented the largest sample sizes were those of Guillemard et al. [33] and Ahrén et al. [22], with 1000 and 898 participants, respectively.

The population of the 18 studies comprised healthy people of both sexes and of working age always greater than 19 years [22-25,27-29,31-39], except for the works of Kinoshita et al. [26], which focused on women, exclusively, Shida et al. [30], which focused on men, exclusively, and Hor et al. [28], which studied an elderly population group. The 
follow-up periods of the studies included in this review ranged from a minimum of 4 weeks [37] to a maximum of 3 years [22].

Most of the selected studies assess the intake of different specific strains of probiotics in the healthy working population and their positive relationship in URTIs. The work of Zhang et al. [23], used a yogurt (Qingrun) with a mixture of Bifidobacterium animalis subsp. lactis Bl-04 ${ }^{\circledR}$, Lacticaseibacillus casei, Lactobacillus delbrueckii bulgaricus, and Streptococcus thermophilus with two plant species used in traditional Chinese medicine, Eriobotrya japonica and Pyrus nivalis.

The species and strains used in the interventions were all non-sporulated Grampositive bacteria, and the concentrations ranged between $10^{6}$ and $10^{11}$ colony-forming units (CFU). There is a current reclassification of the generic term Lactobacilli that reflects the phylogenetic position of microorganisms, and groups lactobacilli into robust clades with shared ecological and metabolic properties [40]. In this way, Lactobacillus plantarum was renamed Lactiplantibacillus plantarum (L. plantarum); Lactobacillus paracasei subsp. paracasei was renamed Lacticaseibacillus paracasei subsp. paracasei (L. paracasei subsp. paracasei); Lactobacillus fermentum was renamed Limosilactobacillus fermentum (L. fermentum); Lactobacillus casei was renamed Lacticaseibacillus casei (L. casei); Lactobacillus rhamnosus was renamed Lacticaseibacillus rhamnosus (L. rhamnosus); and Lactobacillus reuteri was renamed Limosilactobacillus reuteri (L. reuteri).

The different probiotics used in the 18 studies were: L. plantarum HEAL9, L. paracasei subsp. paracasei 8700:2, Streptococcus thermophilus ENT-K12, L. plantarum DR7, Lactobacillus delbrueckii bulgaricus OLL1073R-1, Streptococcus thermophilus, L. paracasei subsp. paracasei, L. casei $431^{\circledR}$, L. fermentum PCC ${ }^{\circledR}$, L. casei Zhang, Bifidobacterium animalis subsp. lactis BB$12^{\circledR}$, L. casei Shirota ${ }^{\circledR}$, L. rhamnosus $\mathrm{LGG}^{\circledR}$, Bifidobacterium animalis subsp. lactis $\mathrm{Bl}-04^{\circledR}$, Bifidobacterium animalis subsp. lactis Bi-07 ${ }^{\circledR} ;$ Lactobacillus acidophilus $\mathrm{NCFM}^{\circledR} ;$ L. rhamnosus LGG $^{\circledR}$; Verum (L. casei DN-114 001, Lactobacillus delbrueckii bulgaricus and Streptococcus thermophilus); Lactobacillus delbrueckii subsp. bulgaricus; Tribion harmonis ${ }^{\mathrm{TM}}$ (Lactobacillus gasseri PA 16/8, Bifidobacterium longum SP 07/3, Bifidobacterium bifidum MF 20/5), and L. reuteri protectis (ATCC55730).

The most used bacterial species, individually or in combination, were B. animalis subsp lactis and L. casei on six occasions and L. plantarum, L. paracasei, and L. delbrueckii subsp. bulgaricus on three occasions each.

In addition to the interventions with these different strains of probiotics, three of the selected studies administered flu vaccines [29,31,32].

In the selected studies, the intake of different probiotic strains in healthy working populations and the impact on upper respiratory tract diseases were analyzed. The results of the different interventions were varied.

The works of Tubelius et al. [35], Berggren et al. [34], and De Vrese et al. [36] demonstrated a significant relationship $(p<0.05)$ between the oral intake of a mixture of probiotics (L. plantarum HEAL9, L. paracasei subsp. paracasei 8700: 2; Tribion harmonis ${ }^{\mathrm{TM}}$; L. reuteri) and a lower incidence of having one or more episodes of the common cold and fewer days of experiencing the symptoms of the common cold. In the study by Ahrén et al. [22] a positive association was demonstrated between two probiotic strains (L. plantarum HEAL9, L. paracasei subsp. paracasei 8700: 2) and protection against colds in adults prone to colds, but without reducing the severity or incidence of colds.

Zhang et al. [23] and Shida et al. [30] concluded that the probiotic mixture Qingrun and L. casei Shirota ${ }^{\circledR}$, respectively, presented a protective effect against URTIs in office workers, reducing the incidence, duration, and severity of the same and improving the immune biomarkers. Recently, the results of Wang et al. [24] in their work on first-line medical personnel during the COVID-19 pandemic (medical staff) stand out. The ingestion of the Streptococcus thermophilus ENT-K12 strain produced a homeostatic relationship between the oropharyngeal microbiota and the cells of the immune system, for at least 20 days. Such a balance could protect medical personnel fighting RTIs, including COVID-19. 
The works by Chong et al. [25] and Hor et al. [28] used a single strain, L. plantarum DR7 and L. casei Zhang, respectively, with similar results in improving the symptoms of URTIs, mainly through the induction of immunomodulatory and anti-inflammatory effects. Additionally, the studies by Guillemard et al. [33] and West et al. [39] significantly associated the incidence of common infections, including respiratory infections, with the ingestion of the probiotic L. casei DN-114 001 and Bifidobacterium animalis subsp. lactis Bl- $04^{\circledR}$, respectively. However, the results of the work of Kinoshita et al. [26] with a probiotic mixture of the strain OLL1073R-1 and Streptococcus thermophilus did not show any prevention of influenza or improvement in the activity of NK cells. However, they did observe a significant increase in the production of $\gamma$-interferon (IFN- $\gamma$ ).

Three studies, by Jespersen et al. [29], Rizzardini et al. [31], and Davidson et al. [32], with L. paracasei subsp. paracasei $431^{\circledR}$ and L. rhamnosus $\mathrm{LGG}^{\circledR}$ and Bifidobacterium animalis subsp. lactis BB-12 ${ }^{\circledR}$, in patients who received the influenza vaccine and live attenuated influenza vaccine (LAIV), presented different results. In the case of L. paracasei subsp. paracasei $431{ }^{\circledR}$, no improvement was noted in the immune response or the concentration of specific antibodies. However, the L. rhamnosus LGG ${ }^{\circledR}$ strain and Bifidobacterium animalis subsp. lactis BB-12 ${ }^{\circledR}$, did improve the immunogenicity of the vaccine and, in addition, the mixture of these two strains in the work of Smith et al. [38] presented a positive association with the Health-Related Quality of Life (HRQoL) perceived by a group of healthy students during the symptoms of URTIs. The results of the work of Meng et al. [37], with the probiotic strain Bifidobacterium animalis subsp. lactis BB-1 ${ }^{\circledR}$, also had a positive impact on the immune system and the severity of URTIs.

\section{Discussion}

The study of the novelty or obsolescence of the chosen topic is quite valid and interesting, since, of all of articles retrieved, approximately $40 \%$ were published in the last 5 years. The Burton Kebler index presented a value in accordance with the expected, while the Price index obtained a slightly higher value in health sciences. However, the Pearson coefficient indicated that the selected articles presented a non-statistically significant increasing linear regression model ( $p=0.33)$ [41].

On the other hand, according to the degree of evidence and recommendations of the US Preventive Services Task Force (USPSTF) [42], controlled and randomized clinical trials (RCTs) are those that provide the most scientific evidence due to their consistent cause-effect relationships. The assessment of the quality of the studies included in this review using CONSORT was acceptable, with a mean of 18.5 out of 25 . Therefore, the grade of recommendation was $B$ (moderate evidence that the measure is effective, and the benefits outweigh the harm).

Likewise, English was the language chosen for the publication of most of the articles since publication in a different language is negative for the impact factor and citations [43]. Furthermore, the number of journals written in English contained in the databases is currently very high [44].

The 18 selected studies focused on URTIs and were carried out on a healthy, working population of men and women, except for the study by Shida et al. [30], which focused exclusively on men, and the work by Kinoshita et al., which was focused exclusively on women. Reinforcing the use of probiotics in other population groups to improve or prevent URTIs, were the works of Makino et al. [45], Shinkai et al. [46] in the elderly population, Wilcox et al. [47] in adults and children, and the study by Tavares-Silva et al. [48] in marathon runners. All probiotic species in the studies analyzed are classified as QPS (qualified presumption of safety). There were hardly any adverse effects in the populations tested.

The fact that in the studies analyzed in this scoping review, 15 different strains of bacteria were used successfully, either alone or in different combinations, suggests that not only that one specific strain or a single combination of probiotics will work, but that many combinations could be a good treatment option. To select the appropriate combination of 
strains, important factors must be considered, such as the fact that not all combinations can obtain positive results. In this sense, the association of Lactobacillus delbrueckii bulgaricus OLL1073R-1 and Streptococcus thermophilus [26] did not show a significant preventive effect against influenza or enhancement in NK lymphocyte activity. It is evident that, in a healthy working population, there are variations between strains in terms of their immunomodulatory capacity on the immune system [22,23,25,27-34,36,37]. The dose used, the duration of the intervention, and seasonal factors may also influence the interpretation of the results [22-39].

In the selected studies, we observed that the genus Lactobacillus [22,23,25-36,38] predominated against Bifidobacterium [23,31,36-39]. The combination of L. plantarum HEAL9 and L. paracasei subsp. paracasei 8700:2 [22,34] and the mixture of L. paracasei subsp. paracasei, L. casei $431{ }^{\circledR}$, and L. fermentum PCC ${ }^{\circledR}[27]$ presented statistically significant favorable results for the biomarker IFN- $\gamma(p=0.045)$ and IFN- $\gamma(p<0.001)$ respectively compared to the placebo group, and in reducing the incidence of URTIs.

Six strains of the genus Lactobacillus, namely L. plantarum DR7, L. casei Zhang, L. casei $431^{\circledR}$, L. casei Shirota ${ }^{\circledR}$, L. rhamnosus LGG ${ }^{\circledR}$, and L. reuteri protectis (ATCC55730), have been used successfully alone in decreasing the risk of contracting URTIs and duration of the symptoms of the common cold [25,28-30,35] as well as in increasing the immunogenicity of an intranasal vaccine against influenza (LAIV) [32]. In agreement with these results are the works of Fonolla et al. [49], Namba et al. [50], and Atkasu et al. [51], although they used different probiotic strains on the elderly population. According to a review by Li et al. [52], which highlights that probiotics induce cell-mediated immunity in phagocytes and natural killer (NK) cells and promote IgA secretion in saliva to enhance vaccine effects, the mechanisms of probiotics in terms of their effects on immune function may be varied. Furthermore, probiotic metabolites, such as short-chain fatty acids, and peptidoglycan components of probiotics appear beneficial to both the host intestinal epithelium and the microbiota by modulating immune function.

The genus Bifidobacterium in our scoping review was always administered in a multistrain probiotic preparation with the genus Lactobacillus [27,31,36-39] or mixed with yogurt smoothies [37]. These combinations with other strains make it almost impossible to assess their specific contribution to the observed effects. However, Bifidobacterium animalis subsp. lactis BB-12 ${ }^{\circledR}$ and Bifidobacterium animalis subsp. lactis Bl-04 ${ }^{\circledR}[37,39]$ demonstrated by themselves a beneficial effect on immunity and reduction in the risk of URTIs in healthy workers.

We highlight the positive results shown with the oropharyngeal probiotic strain Streptococcus thermophilus ENT-K12 [24] used in medical personnel who were in close contact with patients hospitalized for COVID-19, including the formation of a stable microbiota in the oral cavity, protection from respiratory infections for at least 20 days, shorter duration of URTI symptoms, reduced days off due to sick leave, and less use of antibiotics and antivirals. These results should be interpreted with caution, however, because there were some limitations such as non-masking, a small sample size, little follow-up period for medical personnel, etc.

Regarding the use of probiotics in the prevention of COVID-19 in health workers, more studies are currently being carried out to contrast their effectiveness. These are studies that are currently recruiting patients and are registered in the American registry of clinical trials, clinicaltrials.gov (accessed on 4 October 2021) and identified with the codes NCT04366180 and NCT04462627.

The use of preparations with fermented dairy products, such as Qingrun, Verum ${ }^{\circledR}$, and Tribion harmonis ${ }^{\mathrm{TM}}$, could suggest, based on the good results obtained in shift workers [33] and office workers [23], that the combination of strains and species with beneficial effects on the immune system could be a potential nutritional strategy that would address the global problem of respiratory infections and help decrease days off work due to the symptoms caused by URTIs. In accordance with this postulate, Chan et al. [7] in their meta-analysis "Preventing Respiratory Tract Infections by Synbiotic Interventions," proposes a similar 
strategy for improvement of URTIs, in addition to mitigating the misuse of antibiotics used in their treatment.

\section{Limitations}

The results of this review were limited by the shortcomings of each work reviewed [53]. Some commented on the study by Wang et al. [24] or the short follow-up period of 1 month $[24,32,37]$ or the number of people enrolled, [32,37] which could indicate a lack of expected scientific rigor.

Another possible limitation was the small number of articles [22-39] that found and specifically developed an association between the intake of probiotics in working people and RTIs. Furthermore, the population was not homogeneous [28,38], and in some cases the follow-up of the population did not allow the interpretation of favorable results.

\section{Conclusions}

The results of the studies included in this review suggest that probiotics may be a therapeutic tool for public health in URTIs by improving the immune system and reducing days off work. There were practically no adverse effects in the populations tested.

Since probiotics appear to be candidates for the prevention of URTIs and generate significant health savings $[17,18]$, and taking into account the above information, we suggest that a combination including some the probiotics (L. plantarum HEAL9, L. paracasei subsp. paracasei 8700: 2, L. plantarum DR7, L. casei Zhang, L. casei $431^{\circledR}$, L. casei Shirota ${ }^{\circledR}$, L. rhamnosus LGG $^{\circledR}$, Lactobacillus gasseri PA 16/8, L. reuteri, Bifidobacterium animalis subsp. lactis BB-12 ${ }^{\circledR}$ Bifidobacterium animalis subsp. lactis Bl-04 ${ }^{\circledR}$, Bifidobacterium longum SP 07/3, and Bifidobacterium bifidum MF 20/5) should be tested in healthy working populations through clinical trials to evaluate their effectiveness in preventing URTIs and identify the best dosage.

Author Contributions: J.A.P.-M., B.R.-C., P.S.-P., E.N.-D., J.P.-B. and V.N.-L. equally contributed in the preparation of this manuscript, conceptualization, methodology, bibliography data search, information processing, final selection of articles, and data extraction. All authors have read and agreed to the published version of the manuscript.

Funding: This research received no external funding.

Institutional Review Board Statement: Not applicable.

Informed Consent Statement: Not applicable.

Data Availability Statement: Not applicable.

Acknowledgments: P.-M.J.A. thanks to the Mibiopath Reseach Group for their knowledge in the area of microbiota/probiotics and for allowing me to learn with them.

Conflicts of Interest: The authors declare no conflict of interest.

\section{References}

1. Occupational Health [Internet]. Available online: https://www.who.int/westernpacific/health-topics/occupational-health (accessed on 4 October 2021).

2. Benavides, F.G.; Delclós, J.; Serra, C. Estado de bienestar y salud pública: El papel de la salud laboral. Gac. Sanit. 2018, 32, 377-380. [CrossRef] [PubMed]

3. Seguridad y Salud en el Trabajo [Internet]. Available online: https://www.ilo.org/global/standards/subjects-covered-byinternational-labour-standards/occupational-safety-and-health/lang--es/index.htm (accessed on 6 February 2021).

4. Comisión Europea. Marco Estratégico de la UE en Materia de Salud y Seguridad en el Trabajo 2021-2027 [Internet]. Available online: https:/ / eur-lex.europa.eu/legal-content/ES/TXT/HTML/?uri=CELEX:52021DC0323\&from=EN (accessed on 4 October 2021).

5. World Health Organization. WHO I Global Alliance against Chronic Respiratory Diseases [Internet]. Available online: http: //www.who.int/gard/en/ (accessed on 4 October 2021). 
6. User, S. Forum of International Respiratory Societies Releases “The Global Impact of Respiratory Disease" [Internet]. FIRS. Available online: https://www.firsnet.org/news-and-events/news-article/59-forum-of-international-respiratory-societiesreleases-the-global-impact-of-respiratory-disease (accessed on 4 October 2021).

7. Chan, C.K.Y.; Tao, J.; Chan, O.S.; Li, H.-B.; Pang, H. Preventing Respiratory Tract Infections by Synbiotic Interventions: A Systematic Review and Meta-Analysis of Randomized Controlled Trials. Adv. Nutr. 2020, 11, 979-988. [CrossRef] [PubMed]

8. Darbandi, A.; Asadi, A.; Ghanavati, R.; Afifirad, R.; Emamie, A.D.; Kakanj, M.; Talebi, M. The effect of probiotics on respiratory tract infection with special emphasis on COVID-19: Systemic review 2010-20. Int. J. Infect. Dis. 2021, 105, 91-104. [CrossRef]

9. FAO; WHO. Probiotics in Food: Health and Nutritional Properties and Guidelines for Evaluation; FAO Food and Nutrition Paper; Food and Agriculture Organization of the United Nations: Rome, Italy; World Health Organization: Geneva, Switzerland, 2006; 50p.

10. Hill, C.; Guarner, F.; Reid, G.; Gibson, G.R.; Merenstein, D.J.; Pot, B.; Morelli, L.; Canani, R.B.; Flint, H.J.; Salminen, S.; et al. Expert Consensus Document: The International Scientific Association for Probiotics and Prebiotics consensus statement on the scope and appropriate use of the term probiotic. Nat. Rev. Gastroenterol. Hepatol. 2014, 11, 506-514. [CrossRef]

11. Miller, L.E.; Lehtoranta, L.; Lehtinen, M.J. Short-term probiotic supplementation enhances cellular immune function in healthy elderly: Systematic review and meta-analysis of controlled studies. Nutr. Res. 2018, 64, 1-8. [CrossRef] [PubMed]

12. Hajavi, J.; Esmaeili, S.-A.; Varasteh, A.; Vazini, H.; Atabati, H.; Mardani, F.; Momtazi, A.A.; Hashemi, M.; Sankian, M.; Sahebkar, A. The immunomodulatory role of probiotics in allergy therapy. J. Cell. Physiol. 2018, 234, 2386-2398. [CrossRef]

13. Hao, Q.; Dong, B.R.; Wu, T. Probiotics for preventing acute upper respiratory tract infections. Cochrane Database Syst. Rev. 2015, CD006895. [CrossRef] [PubMed]

14. Mahooti, M.; Miri, S.M.; Abdolalipour, E.; Ghaemi, A. The immunomodulatory effects of probiotics on respiratory viral infections: A hint for COVID-19 treatment? Microb. Pathog. 2020, 1, 104452. [CrossRef]

15. Kerry, R.G.; Patra, J.K.; Gouda, S.; Park, Y.; Shin, H.-S.; Das, G. Benefaction of probiotics for human health: A review. J. Food Drug Anal. 2018, 26, 927-939. [CrossRef]

16. Di Stadio, A.; Ishai, R.; Gambacorta, V.; Korsch, F.; Ricci, G.; della Volpe, A.; Bernitsas, E. Nutraceuticals as immune-stimulating therapy to fight COVID-19. Combination of elements to improve the efficacy. Eur. Rev. Med. Pharmacol. Sci. 2020, 24, 9182-9187.

17. Lenoir-Wijnkoop, I.; Gerlier, L.; Bresson, J.-L.; Le Pen, C.; Berdeaux, G. Public Health and Budget Impact of Probiotics on Common Respiratory Tract Infections: A Modelling Study. PLoS ONE 2015, 10, e0122765. [CrossRef]

18. Lenoir-Wijnkoop, I.; Merenstein, D.; Korchagina, D.; Broholm, C.; Sanders, M.E.; Tancredi, D. Probiotics Reduce Health Care Cost and Societal Impact of Flu-Like Respiratory Tract Infections in the USA: An Economic Modeling Study. Front. Pharmacol. 2019, 10, 980. [CrossRef]

19. Wanden-Berghe, C.; Sanz-Valero, J. Systematic reviews in nutrition: Standardized methodology. Br. J. Nutr. 2012, 107 (Suppl. S2), S3-S7. [CrossRef] [PubMed]

20. Moher, D.; Hopewell, S.; Schulz, K.F.; Montori, V.; Gøtzsche, P.C.; Devereaux, P.J.; Elbourne, D.; Egger, M.; Altman, D.G. CONSORT 2010 Explanation and Elaboration: Updated guidelines for reporting parallel group randomised trials. BMJ 2010, 24, 340:c869. [CrossRef]

21. Page, M.J.; McKenzie, J.E.; Bossuyt, P.M.; Boutron, I.; Hoffmann, T.C.; Mulrow, C.D.; Shamseer, L.; Tetzlaff, J.M.; Akl, E.A.; Brennan, S.E. The PRISMA 2020 statement: An updated guideline for reporting systematic reviews. BMJ 2021, $29,372: n 71$. [CrossRef]

22. Ahrén, I.L.; Hillman, M.; Nordström, E.A.; Larsson, N.; Niskanen, T.M. Fewer Community-Acquired Colds with Daily Consumption of Lactiplantibacillus plantarum HEAL9 and Lacticaseibacillus paracasei 8700:2. A Randomized, Placebo-Controlled Clinical Trial. J. Nutr. 2021, 151, 214-222.

23. Zhang, H.; Miao, J.; Su, M.; Liu, B.Y.; Liu, Z. Effect of fermented milk on upper respiratory tract infection in adults who lived in the haze area of Northern China: A randomized clinical trial. Pharm. Biol. 2021, 59, 647-652. [CrossRef]

24. Wang, Q.; Lin, X.; Xiang, X.; Liu, W.; Fang, Y.; Chen, H.; Tang, F.; Guo, H.; Chen, D.; Hu, X.; et al. Oropharyngeal Probiotic ENT-K12 Prevents Respiratory Tract Infections Among Frontline Medical Staff Fighting Against COVID-19: A Pilot Study. Front. Bioeng. Biotechnol. 2021, 9, 646184. [CrossRef]

25. Chong, H.-X.; Yusoff, N.A.A.; Hor, Y.Y.; Lew, L.C.; Jaafar, M.H.; Choi, S.-B.; Yusoff, M.S.; Wahid, N.; Bin Abdullah, M.F.I.L.; Zakaria, N.; et al. Lactobacillus plantarum DR7 improved upper respiratory tract infections via enhancing immune and inflammatory parameters: A randomized, double-blind, placebo-controlled study. J. Dairy Sci. 2019, 102, 4783-4797. [CrossRef]

26. Kinoshita, T.; Maruyama, K.; Suyama, K.; Nishijima, M.; Akamatsu, K.; Jogamoto, A.; Katakami, K.; Saito, I. The effects of OLL1073R-1 yogurt intake on influenza incidence and immunological markers among women healthcare workers: A randomized controlled trial. Food Funct. 2019, 10, 8129-8136. [CrossRef] [PubMed]

27. Zhang, H.; Yeh, C.; Jin, Z.; Ding, L.; Liu, B.Y.; Zhang, L.; Dannelly, H.K. Prospective study of probiotic supplementation results in immune stimulation and improvement of upper respiratory infection rate. Synth. Syst. Biotechnol. 2018, 3, 113-120. Available online: https:/ / www.cochranelibrary.com/central/doi/10.1002/central/CN-01465457/full (accessed on 4 October 2021). [CrossRef] [PubMed]

28. Hor, Y.-Y.; Lew, L.C.; Lau, A.S.-Y.; Ong, J.-S.; Chuah, L.-O.; Lee, Y.Y.; Choi, S.-B.; Rashid, F.; Wahid, N.; Sun, Z.; et al. Probiotic Lactobacillus casei Zhang (LCZ) alleviates respiratory, gastrointestinal \& RBC abnormality via immuno-modulatory, antiinflammatory \& anti-oxidative actions. J. Funct. Foods 2018, 44, 235-245. [CrossRef] 
29. Jespersen, L.; Tarnow, I.; Eskesen, D.; Morberg, C.M.; Michelsen, B.; Bügel, S.; Dragsted, L.O.; Rijkers, G.T.; Calder, P.C. Effect of Lactobacillus paracasei subsp. paracasei, L. casei 431 on immune response to influenza vaccination and upper respiratory tract infections in healthy adult volunteers: A randomized, double-blind, placebo-controlled, parallel-group study. Am. J. Clin. Nutr. 2015, 101, 1188-1196. [CrossRef]

30. Shida, K.; Sato, T.; Iizuka, R.; Hoshi, R.; Watanabe, O.; Igarashi, T.; Miyazaki, K.; Nanno, M.; Ishikawa, F. Daily intake of fermented milk with Lactobacillus casei strain Shirota reduces the incidence and duration of upper respiratory tract infections in healthy middle-aged office workers. Eur. J. Nutr. 2015, 56, 45-53. [CrossRef] [PubMed]

31. Rizzardini, G.; Eskesen, D.; Calder, P.C.; Capetti, A.; Jespersen, L.; Clerici, M. Evaluation of the immune benefits of two probiotic strains Bifidobacterium animalis ssp. lactis, BB- $12{ }^{\circledR}$ and Lactobacillus paracasei ssp. paracasei, L. casei $431^{\circledR}$ in an influenza vaccination model: A randomised, double-blind, placebo-controlled study. Br. J. Nutr. 2011, 107, 876-884. [CrossRef]

32. Davidson, L.E.; Fiorino, A.-M.; Snydman, D.R.; Hibberd, P.L. Lactobacillus GG as an immune adjuvant for live-attenuated influenza vaccine in healthy adults: A randomized double-blind placebo-controlled trial. Eur. J. Clin. Nutr. 2011, 65, 501-507. [CrossRef]

33. Guillemard, E.; Tanguy, J.; Flavigny, A.; De La Motte, S.; Schrezenmeir, J. Effects of consumption of a fermented dairy product containing the probiotic Lactobacillus casei DN-114 001 on common respiratory and gastrointestinal infections in shift workers in a randomized controlled trial. J. Am. Coll. Nutr. 2010, 29, 455-468. [CrossRef]

34. Berggren, A.; Ahrén, I.L.; Larsson, N.; Önning, G. Randomised, double-blind and placebo-controlled study using new probiotic lactobacilli for strengthening the body immune defence against viral infections. Eur. J. Nutr. 2010, 50, 203-210. [CrossRef]

35. Tubelius, P.; Stan, V.; Zachrisson, A. Increasing work-place healthiness with the probiotic Lactobacillus reuteri: A randomised, double-blind placebo-controlled study. Environ. Health Glob. Access Sci. Source 2005, 4, 25. [CrossRef]

36. de Vrese, M.; Winkler, P.; Rautenberg, P.; Harder, T.; Noah, C.; Laue, C.; Ott, S.; Hampe, J.; Schreiber, S.; Heller, K.; et al. Effect of Lactobacillus gasseri PA 16/8, Bifidobacterium longum SP 07/3, B. bifidum MF 20/5 on common cold episodes: A double blind, randomized, controlled trial. Clin. Nutr. Edinb. Scotl. 2005, 24, 481-491. [CrossRef]

37. Meng, H.; Lee, Y.; Ba, Z.; Peng, J.; Lin, J.; Boyer, A.S.; Fleming, J.A.; Furumoto, E.J.; Roberts, R.F.; Kris-Etherton, P.M.; et al. Consumption of Bifidobacterium animalis subsp. lactis BB-12 impacts upper respiratory tract infection and the function of NK and T cells in healthy adults. Mol. Nutr. Food Res. 2016, 60, 1161-1171. [CrossRef]

38. Smith, T.J.; Rigassio-Radler, D.; Denmark, R.; Haley, T.; Touger-Decker, R. Effect of Lactobacillus rhamnosus LGG ${ }^{\circledR}$ and Bifidobacterium animalis ssp. lactis BB- $12^{\circledR}$ on health-related quality of life in college students affected by upper respiratory infections. Br. J. Nutr. 2013, 109, 1999-2007. [CrossRef]

39. West, N.P.; Horn, P.L.; Pyne, D.; Gebski, V.; Lahtinen, S.J.; Fricker, P.A.; Cripps, A.W. Probiotic supplementation for respiratory and gastrointestinal illness symptoms in healthy physically active individuals. Clin. Nutr. 2014, 33, 581-587. [CrossRef]

40. Zheng, J.; Wittouck, S.; Salvetti, E.; Franz, C.M.A.P.; Harris, H.M.B.; Mattarelli, P.; O'Toole, P.W.; Pot, B.; Vandamme, P.; Walter, J.; et al. A taxonomic note on the genus Lactobacillus: Description of 23 novel genera, emended description of the genus Lactobacillus Beijerinck 1901, and union of Lactobacillaceae and Leuconostocaceae. Int. J. Syst. Evol. Microbiol. 2020, 70, 2782-2858. [CrossRef]

41. Franco-López, A.; Sanz-Valero, J.; Culebras, J.M. El factor de impacto ya no es el patrón oro; la declaración de San Francisco sobre evaluación de la investigación. J. Negat. No Posit. Results JONNPR 2017, 2, 173-176.

42. Harris, R.P.; Helfand, M.; Woolf, S.H.; Lohr, K.N.; Mulrow, C.D.; Teutsch, S.M.; Atkins, D.; Methods Work Group. Third US Preventive Services Task Force Current methods of the U.S. Preventive Services Task Force: A review of the process. Am. J. Prev. Med. 2001, 20, 21-35. [CrossRef]

43. López, Á.F.; Valero, J.S.; Fernández, J.M.C. Publicar en castellano, o en cualquier otro idioma que no sea inglés, negativo para el factor de impacto y citaciones. J. Negat. No Posit. Results JONNPR 2016, 1, 65-70.

44. Melián-Fleitas, L.; Franco-Pérez, Á.M.; Sanz Valero, J. Análisis bibliométrico y temático de la producción científica sobre salud laboral relacionada con nutrición, alimentación y dieta, indexada en MEDLINE. Med. Segur. Trab. 2019, 65, 10-23. [CrossRef]

45. Makino, S.; Ikegami, S.; Kume, A.; Horiuchi, H.; Sasaki, H.; Orii, N. Reducing the risk of infection in the elderly by dietary intake of yoghurt fermented with Lactobacillus delbrueckii ssp. bulgaricus OLL1073R-1. Br. J. Nutr. 2010, 104, 998-1006. [CrossRef] [PubMed]

46. Shinkai, S.; Toba, M.; Saito, T.; Sato, I.; Tsubouchi, M.; Taira, K.; Kakumoto, K.; Inamatsu, T.; Yoshida, H.; Fujiwara, Y.; et al. Immunoprotective effects of oral intake of heat-killed Lactobacillus pentosus strain b240 in elderly adults: A randomised, double-blind, placebo-controlled trial. Br. J. Nutr. 2012, 109, 1856-1865. [CrossRef] [PubMed]

47. Wilcox, C.; Stuart, B.; Leaver, H.; Lown, M.; Willcox, M.; Moore, M.; Little, P. Effectiveness of the probiotic Streptococcus salivarius K12 for the treatment and/or prevention of sore throat: A systematic review. Clin. Microbiol. Infect. 2019, 25, 673-680. [CrossRef] [PubMed]

48. Tavares-Silva, E.; Caris, A.; Santos, S.; Ravacci, G.; Thomatieli-Santos, R. Effect of Multi-Strain Probiotic Supplementation on URTI Symptoms and Cytokine Production by Monocytes after a Marathon Race: A Randomized, Double-Blind, Placebo Study. Nutrients 2021, 13, 1478. [CrossRef]

49. Fonollá, J.; Gracián, C.; Maldonado-Lobón, J.A.; Romero, C.; Bédmar, A.; Carrillo, J.C.; Martín-Castro, C.; Cabrera, A.L.; GarcíaCuriel, J.M.; Rodríguez, C.; et al. Effects of Lactobacillus coryniformis K8 CECT5711 on the immune response to influenza 
vaccination and the assessment of common respiratory symptoms in elderly subjects: A randomized controlled trial. Eur. J. Nutr. 2017, 58, 83-90. [CrossRef]

50. Namba, K.; Hatano, M.; Yaeshima, T.; Takase, M.; Suzuki, K. Effects of Bifidobacterium longum BB536 administration on influenza infection, influenza vaccine antibody titer, and cell-mediated immunity in the elderly. Biosci. Biotechnol. Biochem. 2010, 74, 939-945. [CrossRef] [PubMed]

51. Akatsu, H.; Nagafuchi, S.; Kurihara, R.; Okuda, K.; Kanesaka, T.; Ogawa, N.; Kanematsu, T.; Takasugi, S.; Yamaji, T.; Takami, M.; et al. Enhanced vaccination effect against influenza by prebiotics in elderly patients receiving enteral nutrition. Geriatr. Gerontol. Int. 2015, 16, 205-213. [CrossRef] [PubMed]

52. Lei, W.-T.; Shih, P.-C.; Liu, S.-J.; Lin, C.-Y.; Yeh, T.-L. Effect of Probiotics and Prebiotics on Immune Response to Influenza Vaccination in Adults: A Systematic Review and Meta-Analysis of Randomized Controlled Trials. Nutrients 2017, 9, 1175. [CrossRef]

53. Domingo-Pueyo, A.; Sanz-Valero, J.; Wanden-Berghe, C. Efectos sobre la salud de la exposición laboral al cromo y sus compuestos: Revisión sistemática-[Effects of occupational exposure to chromium and its compounds: A systematic review]. Arch. Prev. Riesgos Labor. 2014, 17, 142-153. [CrossRef] [PubMed] 